\title{
CORRECTION
}

\section{Correction to: Effect of the WC Composition Ratio on the Microstructure and Properties of WC-10Ni+AgCuTi Coatings}

Xiangping Xu, Chi Liu, Chunzhi Xia, Yi Wang, Jiasheng Zou, and Xiaoguo Song

/ Published online: 7 February 2022

\section{Correction to: JMEPEG}

\section{https://doi.org/10.1007/s11665-022-06581-2}

In this published article, the corresponding author was identified incorrectly, and an affiliation was omitted. The corresponding author is Chunzhi Xia, and the correct list of affiliations is shown below:

Xiangping Xu, Chi Liu, Yi Wang, and Jiasheng Zou, School of Materials Science and Engineering, Jiangsu University of Science and Technology, Zhenjiang 212003, People's Republic of China; Chunzhi Xia, School of Materials Science and Engineering, Jiangsu University of Science and Technology,
Zhenjiang 212003, People's Republic of China; and State Key Laboratory of Advanced Welding and Joining, Harbin Institute of Technology, Harbin 150000, People's Republic of China; and Xiaoguo Song, State Key Laboratory of Advanced Welding and Joining, Harbin Institute of Technology, Harbin 150000, People's Republic of China. Contact e-mail: cz_xia@126.com.

Publisher's Note Springer Nature remains neutral with regard to jurisdictional claims in published maps and institutional affiliations.

The original article can be found online at https://doi.org/10.1007/ s11665-022-06581-2.

Xiangping Xu, Chi Liu, Yi Wang, and Jiasheng Zou, School of Materials Science and Engineering, Jiangsu University of Science and Technology, Zhenjiang 212003, People's Republic of China; Chunzhi Xia, School of Materials Science and Engineering, Jiangsu University of Science and Technology, Zhenjiang 212003, People's Republic of China; and State Key Laboratory of Advanced Welding and Joining, Harbin Institute of Technology, Harbin 150000, People's Republic of China; and Xiaoguo Song, State Key Laboratory of Advanced Welding and Joining, Harbin Institute of Technology, Harbin 150000, People's Republic of China. Contact e-mail: cz_xia@126.com. 